\title{
Karitative Hilfe für Solidarność
}

\author{
Dariusz Wojtaszyn
}

Im Jahr 2010 wurden von Europejskie Centrum Solidarności in Danzig die Medaille der Dankbarkeit an 326 Personen aus der ganzen Welt verteilt. Die Mehrheit der ausgezeichneten Personen kommt aus Frankreich (135), aber auf der zweiten Stelle sind die deutschen Bürger - insgesamt 46 Personen aus der ehemaligen Bundesrepublik und der DDR, die Solidarność in den achtziger Jahren unterstützten. ${ }^{1}$

Die Hilfe für Solidarność zu leisten war in den achtziger Jahren keine einfache Aufgabe in beiden deutschen Staaten. Es war mit der politischen Situation verbunden - die Regierungen beider deutschen Staaten - trotz aller Unterschiede - beunruhigte die Situation in Polen, beide waren an einer Beruhigung interessiert. Die Gründung der Solidarność entsprach nicht der Idee der sozialliberalen, sogenannten „Neuen Ostpolitik“" , die - in den Beziehungen zu den Ländern des kommunistischen Blocks - auf der Strategie der Entspannungspolitik und dem allmählichen Aufweichen ihres Kurses fußte. Diese Politik, die zwar in mehreren Bereichen Erfolge brachte, berücksichtigte aber nicht den wichtigen gesellschaftlichen Faktor- die polnische politische Opposition. ${ }^{3}$

${ }^{1}$ Vgl. Barbara Cöllen. „Medal Wdzięczności“ - Solidarność dziękuje 46 Niemcom, http://www.dw-world.de/dw/article/0,,5954571,00.html (letzter Zugriff 02.10.2011); andere ausgezeichnete Personen kamen aus Italien, Großbritannien, Norwegen, Schweden, Australien, USA, Belgien, Portugal, der Schweiz, der Slowakei und den Niederlanden.

${ }^{2}$ Vgl. z. B. Katarzyna Stokłosa, Polen und die deutsche Ostpolitik 1945-1990, Göttingen 2011; Carole Fink/Bernd Schaefer (Hgg.), Ostpolitik, 1969-1974. European and Global Responses, Cambridge 2009.

${ }^{3}$ Symptomatisch ist Egon Bahrs Meinung, einem Vordenker und Architekten der „Neuen Ostpolitik“, der gestand: „Wir trauten Solidarnosc nicht das Augenmaß zu, die Sehne nicht zu überspannen. Das war ebenso falsch wie die Annahme, dass ein kommunistisch regiertes Land im Block nicht von unten, sondern nur von oben veränderbar sei [...] Wir haben Solidarnosc unterschätzt und nicht ernst genug genommen.“, Egon Bahr, Zu meiner Zeit, München 1996, 343; vgl. auch Dieter Bingen, Polityka Republiki Bońskiej

KZG/CCH 24 (2011), S. 1-10, ISSN 0932-9951

(C) Vandenhoeck \& Ruprecht GmbH \& Co. KG, Göttingen 2012 
Die neue Rolle der polnischen Opposition in der Solidarność-Ära bedeutete für die westdeutsche Regierung ein Dilemma: Was heißt gute Nachbarschaft mit Polen, mit welchen Kräften soll man solidarisch sein? In Bonn musste man auch das deutsch-deutsche Verhältnis berücksichtigen. Eine Priorität für die sozialliberale Bundesregierung war die Erhaltung der humanitären Erleichterungen für manche Bürger der DDR - auch um den Preis der Stabilisierung des SED-Regimes - sie durfte darum nicht zulassen, dass die politischen Kontakte und Solidarität mit der polnischen Demokratiebewegung zu Problemen im Verhältnis mit der DDR führten. ${ }^{4}$ Darum war die Reaktion der Bundesregierung auf die polnische Freiheitsbewegung - nach Timothy Garton Ash - extrem defensiv und kritisch. ${ }^{5}$ Kein Vertreter der Regierung erwähnte offiziell die Entwicklung in der Volksrepublik Polen, um den Vorwurf der Einmischung in die inneren Angelegenheiten Polens zu vermeiden. ${ }^{6}$

Auch die Reaktion auf die Verhängung des Kriegsrechts in Polen war zurückhaltend und vorsichtig. Bundeskanzler Helmut Schmidt war gerade in diesen Tagen (11-13. Dezember 1981) zu offiziellem Besuch in Ost-Berlin (etwa 80 Kilometer von Polens-Grenze entfernt), wo er keine aufsehenerregende Geste machen wollte und den Besuch reibungslos fortsetzte. ${ }^{7}$ Die

wobec Polski. Od Adenauera do Kohla 1949-1991, Kraków 1997, 193 ff.; Jerzy Maćków, Die Entspannungspolitik der Bundesrepublik Deutschland gegenüber der Entwicklung in Polen in den siebziger und achtziger Jahren, in: Zeitschrift für Politik, Nr. 4, 1993, 372 ff; Timothy Garton Ash, Im Namen Europas. Deutschland und der geteilte Kontinent, Frankfurt/M. 1995, 268; Artur Hajnicz, Polens Wende und Deutschlands Vereinigung. Die Öffnung zur Normalität 1989-1992, Paderborn 1995, 31.

${ }^{4}$ Bingen, Polityka Republiki, aaO. (Anm. 3), 195 f.; ders., Die politischen Beziehungen zwischen der Bundesrepublik Deutschland und Polen (1975-1991), in: Die lange Nachkriegszeit. Deutschland und Polen von 1945 bis 1991, Schriftenreihe des Georg-EckertInstituts für internationale Schulbuchforschung, Band 22/XIV, Braunschweig 1995, 126; Maćków, Entspannungspolitik, aaO. (Anm. 3), 372 ff.

${ }^{5}$ Ash, Im Namen Europas, aaO. (Anm. 3), 268.

${ }^{6}$ Bingen, Polityka Republiki, aaO. (Anm. 3), 196.

${ }^{7}$ H. Schmidt reagierte auf die Information über die Verhängung des Kriegsrechts in Polen mit den Wörtern: „Herr Honecker ist genauso bestürzt wie ich, dass dies nun notwendig war", Helmut Schmidt, Die Deutschen und ihre Nachbarn, Menschen und Mächte, Berlin 1990, 74; vgl. Dariusz Wojtaszyn, Obraz Polski i Polaków w prasie i literaturze Niemieckiej Republiki Demokratycznej w okresie powstania Solidarności i stanu wojennego, Wrocław 2007, 69-70; Manfred Wilke/Reinhardt Gutsche/Michael Kubina, Die SED-Führung und die Unterdrückung der polnischen Oppositionsbewegung 1980/81, Bericht des BIOst, Nr 36/1994, Köln 1994, 30, 106.

KZG/CCH 24 (2011), S. 1-10, ISSN 0932-9951

(C) Vandenhoeck \& Ruprecht GmbH \& Co. KG, Göttingen 2012 
BRD-Regierung hat darüber hinaus keine wirtschaftlichen Sanktionen für die Volksrepublik Polen verkündet, was einen Streit mit der Verwaltung des neuen amerikanischen Präsident Ronald Reagan verursachte. ${ }^{8}$ Auch für die Bundesbürger waren die Ziele und Tätigkeiten der Solidarność nicht völlig verständlich.

In der DDR verfolgte man die politischen Ereignisse in Polen der Jahre 1980-1981 mit Unruhe und Argwohn. Die politische Krise beim Nachbarn gefährdete - wie man annahm - die Stabilität und Festigkeit des sozialistischen Lagers und somit die der DDR. ${ }^{9}$ Die Staatsmacht der DDR fürchtete den „Virus der Solidarnośćc im eigenen Lande. Deswegen betrieb die SEDFührung eine Isolierungspolitik gegenüber Polen; auch die DDR-Bürger wurden von den Informationen aus und über Polen abgeschnitten. ${ }^{10}$ Darüber hinaus gehörte die SED-Führung zu den entschiedenen Befürwortern einer militärischen Intervention der Armeen des Warschauer Paktes in Polen. ${ }^{11}$

Außerhalb der offiziellen Politik gab es aber viele Reaktionen einzelner Personen, gesellschaftlicher Gruppen und Institutionen in beiden deutschen Staaten auf die polnische Solidarność-Revolution und die Verhängung des Kriegsrechts in Polen.

\footnotetext{
${ }^{8}$ Bingen, Polityka Republiki, aaO. (Anm. 3), 205 ff..

${ }^{9}$ Die Ereignisse in Polen wurden oft als „Konterrevolution“ bezeichnet. Zum ersten Mal wurde der Begriff am 31.08.1980 vom DDR-Botschafter in Warschau, Günther Sieber, in seinem Bericht benutzt, vgl. Die politische Entwicklung der VR Polen zwischen dem 4. und 5. Plenum des ZK der PVAP (Woche vom 24. bis 30. 08.) und die Lage am 31.08.1980, in: Michael Kubina/Manfred Wilke (Hgg.), „Hart und kompromisslos durchgreifen“. Die SED contra Polen 1980/81. Geheimakten der SED-Führung über die Unterdrückung der polnischen Demokratiebewegung, Berlin 1995, 48-56.

${ }^{10}$ Die DDR-Regierung kündigte am 30.10.1980 einseitig den Visa- und passfreien Reiseverkehr zwischen der DDR und der VR Polen, vgl. Burkhard Olschowsky, Einvernehmen und Konflikt. Das Verhältnis zwischen der DDR und der Volksrepublik Polen 1980-1989, Osnabrück 2005, 37; Wojtaszyn, Obraz Polski, aaO. (Anm. 7), 74.

${ }^{11}$ Vgl. z. B. Brief E. Honecker an L. Brežnev vom 26. November 1980, in: Kubina/Wilke, Hart und kompromißlos durchgreifen, aaO. (Anm. 9), 122; Stenografische Niederschrift des Treffens führender Repräsentanten der Teilnehmerstaaten des Warschauer Vertrages am 5. Dezember 1980 in Moskau, in: Ebd., 166; Äußerungen der Genossen E. Honecker und St. Kania während der Fahrt am 17.02.1981 nach und von Hubertusstock, in: Ebd., 223; Gespräch des Generalsekretärs des ZK der SED und Vorsitzenden des Staatsrates der DDR, Erich Honecker, anlässlich seines Aufenthaltes in Kuba mit dem Ersten Sekretär des ZK der KP Kubas und Vorsitzenden des Staatsrates und des Ministerrates der Republik Kuba Fidel Castro, am 13. September 1981 in Havanna, in: Ebd., 340-342.
}

KZG/CCH 24 (2011), S. 1-10, ISSN 0932-9951

(c) Vandenhoeck \& Ruprecht GmbH \& Co. KG, Göttingen 2012 
Die wichtige Unterstützung für Solidarność aus der Bundesrepublik kam seit Dezember 1980 seitens des Deutschen Gewerkschaftsbunds (DGB), der praktische Solidarität zu üben versuchte. DGB-Vertreter wollten die oft unbekannten politischen Ideen der polnischen politischen Opposition in die Öffentlichkeit der Bundesrepublik tragen und eine öffentliche Debatte über die Situation in Polen fördern. Die Gewerkschaftler unterstützten Solidarność in ihrer Arbeit vor allem auch materiell. ${ }^{12}$

In verschiedenen deutschen Städten entstanden Solidaritäts-Komitees, die versuchten mit öffentlichen Veranstaltungen, Pressesammlungen, Informationsbroschüren, aber auch Radio- und Fernsehbeiträgen Aufmerksamkeit für die Solidarność in der BRD zu wecken. ${ }^{13}$

Nach den alarmierenden Berichten der westdeutschen Medien im Jahr 1981 über die politische Situation in der Volksrepublik Polen und vor allem über die katastrophale Lage der polnischen Bürger ${ }^{14}$, besonders durch die Versorgungsprobleme, kam es in verschiedenen Städten zu ersten spontanen Aktionen für Lebensmittel- und Kleiderspenden, die dann von Privatpersonen, kommerziellen Sammelstellen (gegen ein nicht zu hohes Entgelt) oder der Bundespost an verschiedene Zielorte nach Polen transportiert wurden. ${ }^{15}$

An den Spenden beteiligten sich vor allem private Personen, es wurden aber auch das Deutsche Rote Kreuz ${ }^{16}$ und kirchliche Institutionen tätig. Eine bedeutende Rolle spielte hier der Katholische Deutsche CaritasVerband. Caritas organisierte seit Anfang 1981 viele Gütertransporte nach Polen - zu diesem Zweck kaufte man sogar vier Lastkraftwagen. ${ }^{17} \mathrm{Zu}$ den

\footnotetext{
${ }^{12}$ Natalie Begin, Kontakte zwischen Gewerkschaften in Ost und West. Die Auswirkungen von Solidarność in Deutschland und Frankreich. Ein Vergleich, in: Archiv für Sozialgeschichte, Band 45, 2005, 297 ff.

${ }^{13}$ Andrea Genest, Die Solidarność aus deutscher Perspektive, in: Potsdamer Bulletin für Zeithistorische Studien, Nr. 34/35, 2005, 19.

${ }^{14}$ Vgl. z. B. Katastrophe in Sicht, in: Der Spiegel, Nr. 47, 1981, 142-144; Die Machtfrage ist gestellt. Polens Vizepremier Mieczyslaw Rakowski über die Staatskrise, in: Der Spiegel, Nr. 38, 1981, 142-143; Ein General regiert in Polen, in: Der Spiegel, Nr. 8, 1981, 124-129.

${ }^{15}$ Albrecht Riechers, Hilfe für Solidarność. Zivilgesellschaftliche und staatliche Beispiele aus der Bundesrepublik Deutschland in den Jahren 1980-1982, Bonn 2006, 17.

${ }^{16}$ Vgl. Polen-Hilfe: Eine echte Volksbewegung, in: Der Spiegel, Nr. 23, 1982, 83.

${ }^{17}$ Barbara Cöllen, Pomoc niemieckiego Caritasu, in: Polenhilfe: Als Schmuggler unterwegs (im Druck).
}

KZG/CCH 24 (2011), S. 1-10, ISSN 0932-9951

(C) Vandenhoeck \& Ruprecht GmbH \& Co. KG, Göttingen 2012 
größten Caritas-Spendern gehörte das Ruhrgebiet. ${ }^{18}$ Ein weiteres Beispiel ist der 1981 entstandene, jahrelange Austausch zwischen den katholischen Gemeinden in Dortmund und Wrocław. ${ }^{19}$ Die Katholiken aus Dortmund leisteten zunächst materielle Hilfe, unternahmen später aber den Versuch, viele Kontakte in Oberschlesien aufzubauen, die sich mittlerweile zu langfristigen Freundschaften entwickelt haben.

Die Verhängung des Kriegsrechts in der Volksrepublik Polen rief eine moralische Empörung in breiten Kreisen der Gesellschaft der BRD hervor. Es fanden landesweite Sympathiekundgebungen für Solidarność statt. Diese Situation mobilisierte die westdeutsche Bevölkerung zu intensiver Aktivität und rief eine große Hilfsaktion hervor.

Schon wenige Tage vor dem 13. Dezember 1981 beschloss der DGB die Hilfsaktion „Solidarität für Polen, Deutscher Gewerkschaftsbund“ ins Leben zu rufen. Nach der Verhängung des Kriegsrechts in Polen gründete der DGB einen offiziellen Verein - Solidarität für Polen e.V. der bis März 1982 1,7 Millionen Mark sammelte. ${ }^{20}$ Sie schickten auch die Lebensmitteltransporte in die Volksrepublik Polen. Die Hilfsaktionen des DGB liefen alle über etablierte karitative Organisationen, wie das Deutsche Rote Kreuz, weil die polnischen Behörden direkte Lieferungen des DGB nicht gestatteten. Die Spendenmittel wurden dann von der polnischen katholischen Kirche - als der einzigen nicht-staatlichen Organisation, die in Polen funktionierte - verteilt. $^{21}$

Breite Kreise der BRD-Bevölkerung beteiligten sich an den PaketeAktionen, die zu den bedeutsamsten Unterstützungsmaßnahmen in der BRD gehörten. Der Umfang der spontanen Hilfe für Polen war sehr groß. Angetrieben von menschlichen, aber gelegentlich auch antikommunistischen Gefühlen ${ }^{22}$, sammelten und spendeten für Polen Institutionen,

\footnotetext{
${ }^{18}$ Ebd.

${ }^{19}$ Andreas Bornholdt, Solidarität von Gemeinde zu Gemeinde und Schule zu Schule. Breslau-Dortmund 1981-1989. Ein Beitrag zur Völkerverständigung und Versöhnung zwischen Deutschen und Polen, Dortmund 1990.

${ }^{20}$ Begin, Kontakte zwischen Gewerkschaften, aaO. (Anm. 12), 302.

${ }^{21}$ Ebd., 302-303; Małgorzata Świder, Zwischenmenschlichen Brücken. Deutsche „Hilfe für Solidarnośćc als Beispiel der deutsch-polnischen Beziehungen in den achtziger Jahren, in: Brücke zwischen den Kulturen. Geistige Grundlage. Historische Beispiele. Zeitfragen, Frankfurt/M. 2009, $231 \mathrm{ff}$.

${ }^{22}$ Der Spiegel, Polen-Hilfe, aaO. (Anm. 16), 82-83.
}

KZG/CCH 24 (2011), S. 1-10, ISSN 0932-9951

(c) Vandenhoeck \& Ruprecht GmbH \& Co. KG, Göttingen 2012 
Organisationen, Parteien, verschiedene Verbände und Vereine, Firmenbelegschaften und Schulkinder, aber vor allem private Personen. Diese Aktion wurde auch von der staatlichen Seite gefördert. Der Bundestag hatte eine befristete Gebührenbefreiung für Postpakete nach Polen beschlossen, die am 8. Februar 1982 in Kraft trat. Die erste Gebührenbefreiung dauerte bis zum 30. Juni 1982 und bewirkte ein Ansteigen der Anzahl eingelieferter Pakete - nach den Angaben der Deutschen Post um etwa 600\% ${ }^{23}$ - etwa 35.000 Geschenkpakete pro Tag. ${ }^{24}$

Die Spenden-Aktion lief aber nicht ohne kleine Schwierigkeiten ab. Abgesehen von logistischen Problemen, wurden Gerüchte verbreitet, dass die Hilfe nicht zu den richtigen Personen gelangte. Die Spender befürchteten, dass zu den Empfängern der Pakete vor allem Partei-, Armee- Miliz- und Sicherheitsangehörige gehörten. ${ }^{25}$ Um diese Ängste zu überwinden wurde von der Post eine spezielle Liste von Empfangsstellen in Polen vorbereitet, an die ab dem 1. März 1982 portofreie Pakete geschickt werden konnten. Zu den Empfängern gehörten: das Polnische Rote Kreuz, das Polnische Komitee für Soziale Hilfe in Warschau, Danzig, Posen und Kattowitz, die Gesellschaft der Freunde des Kindes in Warschau, Krakau, Allenstein, Breslau, Lublin und Piła, der Caritas-Verband der Katholiken Polens in Warschau, Kattowitz, Posen, Słupsk (Stolp), das karitative Komitee des Polnischen Episkopats in Kattowitz, Warschau, Posen, Breslau und Krakau und der Polnische Ökumenische Rat in Warschau. ${ }^{26}$

Nach der Zeit der wieder aufgenommenen Gebührenpflicht wurde die Zahl der eingelieferten Pakete verringert. Ab Anfang Juli 1982 sank die Zahl der Sendungen ${ }^{27}$ auf 4.000 bis 5.000 Stück pro Tag (was wahrscheinlich auch durch die Sommerferien bedingt war). Die Vertreter der Deutsch-Polnischen

\footnotetext{
${ }^{23}$ Riechers, Hilfe für Solidarność, aaO. (Anm. 15), 20.

${ }^{24}$ Der Spiegel, Polen-Hilfe, aaO. (Anm. 16), 82

${ }^{25}$ Riechers, Hilfe für Solidarność, aaO. (Anm. 15), 19; Der Spiegel, Polen-Hilfe, aaO. (Anm. 16), 87-90; sogar polnische politische Emigranten (u.a. Leszek Kołakowski und Czesław Miłosz) hatten einen „Polnischen Appell an die Deutschen“ eingereicht (3. Mai 1982), in dem sie forderten: „Humanitäre Hilfe für die notleidende polnische Bevölkerung muss außerhalb jeglicher Regierungskanäle verlaufen - nur über kirchliche und unabhängige karitative Organisationen oder vertrauenswürdige Einzelpersonen.", ebd., 90. Ähnliche Befürchtungen waren auch in der DDR-Bevölkerung verbreitet, s. BStU MfS ZAIG 4152, 53; BStU MfS HA VII 2959/Bd. 4, 24.

${ }^{26}$ Riechers, Hilfe für Solidarność, aaO. (Anm. 15), 19

${ }^{27}$ Vgl. Ebd., 21; Der Spiegel, Polen-Hilfe, aaO. (Anm. 16), 82 ff.
} 
Gesellschaften ${ }^{28}$ und manche Politiker (u. a. der Bundeskanzler) ${ }^{29}$ riefen die BRD-Bürger auf, die Hilfe für Polen - trotz der Porto-Pflicht - wegen der Gefahr der Hungersnot der polnischen Bevölkerung nicht auszulassen und fortzusetzen. Die Appelle hatten Erfolg - im Juli 1982 betrug die Zahl der nach Polen geschickten Pakete 60.000, im August 86.000, im September 106.000 und im Oktober gar 110.000. ${ }^{30}$

Gleichzeitig folgten politische Veränderungen in der Bundesrepublik. Am 1. Oktober kam es zum Regierungswechsel - der neue Bundeskanzler wurde Helmut Kohl (CDU). ${ }^{31}$ Die neue Regierung beteiligte sich auch an der Hilfsaktion - sie initiierte eine Wiederaufnahme der gebührenfreien Versendung nach Polen, die ab 1. November 1982 bis zum Jahresende dauerte. Im gesamten gebührenfreien Zeitraum (sieben Monate: Februar-Juni; November-Dezember 1982) wurden aus dem Gebiet der Bundesrepublik etwa 8,6 Millionen ${ }^{32}$ Sendungen nach Polen geschickt. Nach den Angaben der Bundespost kostete die Hilfsaktion für Polen den bundesdeutschen Steuerzahler 174,8 Millionen Mark ${ }^{33}$ - das Auswärtige Amt hat diese Summe (der BRD-Post, der polnischen Post und auch für den Transit der DDR-Post) erstattet.

Die Hilfsaktion für Polen dauerte aber trotz des wieder zu entrichtenden Paketportos weiter an - im Jahr 1983 schickten die Bundesbürger etwa 2 Millionen und 1984 etwa 1,5 Millionen Sendungen nach Polen. ${ }^{34}$ Bei der Berücksichtigung aller bekannten Zahlen (gebührenpflichtige und gebührenfreie Pakete) betrug die Summe etwa 14,4 Millionen Sendungen. ${ }^{35}$

Die großangelegte Polenhilfe war beispiellos. Die Publizisten des Nachrichtenmagazins „Der Spiegel“ haben sie als „echte Volksbewegung“ bezeichnet. In der Zeitschrift war folgende Beurteilung zu lesen:

\footnotetext{
${ }^{28}$ Riechers, Hilfe für Solidarność, aaO. (Anm. 15), 21.

${ }^{29}$ Der Spiegel, Polen-Hilfe, aaO. (Anm. 16), 82.

${ }^{30}$ Riechers, Hilfe für Solidarność, aaO. (Anm. 15), 21-22.

${ }^{31}$ Über Politik der CDU/CSU-FDP-Regierung zu Polen s. z. B. Bingen, Polityka Republiki, aaO. (Anm. 3), $213 \mathrm{ff}$.

${ }^{32}$ Riechers, Hilfe für Solidarność, aaO. (Anm. 15), 22.

${ }^{33}$ Vgl. ebd. 22-23.

${ }^{34}$ Ebd. 23-24.

${ }^{35}$ Ebd. 24.

KZG/CCH 24 (2011), S. 1-10, ISSN 0932-9951

(c) Vandenhoeck \& Ruprecht GmbH \& Co. KG, Göttingen 2012
} 
„Noch nie haben die Wohlstandsbürger so viel, so freizügig, so ausdauernd gespendet, noch nie waren sie so aktiv, selber Spenden zu sammeln und Hilfsaktionen zu organisieren. Sie opferten ihre Freizeit [...] sie entwickelten Phantasie [...]."36

Auch Politiker haben die Hilfsaktionen mit Freude begrüßt. Der Bundestag hat sie in einer Resolution als Bekundung der „Mitmenschlichkeit und moralischen Solidarität ${ }^{“{ }^{37}}$ bezeichnet und der damalige Außenminister HansDietrich Genscher fügte hinzu: „Die spontane Hilfsbereitschaft unser Bürger ist schon beeindruckend“" ${ }^{38}$

Teilweise unbekannt ist die Tatsache, dass an der Hilfsaktion für Polen auch die DDR teilnahm. ${ }^{39}$ Am 18. Dezember 1981 verkündete die DDR-Regierung durch das Präsidium des ostdeutschen Roten Kreuzes und die Organisation Volkssolidarität Vermittlung Aktionen „Hilfe für die Volksrepublik Polen“ und „Hilfe für Kinder Volkspolens“. ${ }^{40}$ Im Rahmen der Aktionen wurden die Lebensmittel-, Kleider- und Arzneimittel-Spenden gesammelt; es wurde auch ein Sonderkonto „Konto 555“ vorbereitet, an das die DDR-Bürger Geld einzahlen konnten. Ähnliche Aktionen initiierten auch andere in der DDR funktionierende Organisationen - z. B. die Freie Deutsche Jugend und die Pionierorganisation „Ernst Thälmann“, die 2,5 Millionen Weihnachtspakete für polnische Schüler in den DDR-Schulen vorbereiteten und nach Polen schickten. ${ }^{41}$

Die SED-Leitung betrachtete die von außen vorbereiteten Hilfsmaßnahmen für Polen vor allem als eine Propaganda-Aktion. Es sollte ein Zeichen der Solidarität sein, aber nicht der Solidarität mit der notleidenden polnischen Nation, sondern mit der polnischen kommunistischen Partei - der Polnischen Vereinigten Arbeiterpartei (PVAP), die endlich den von der SED erwarteten harten Kurs gegenüber Solidarność präsentierte. Die Hilfe aus

\footnotetext{
${ }^{36}$ Der Spiegel, Polen-Hilfe, aaO. (Anm. 16), 82.

${ }^{37}$ Ebd., 82.

${ }^{38}$ Ebd., 83.

${ }^{39}$ Mehr dazu s. Dariusz Wojtaszyn, Akcja pomocy NRD dla Polski po ogłoszeniu stanu wojennego - odruch serca czy przedsięwzięcie propagandowe, in: Przyjaźń nakazana? Stosunki między NRD i Polską w latach 1949-1990, red. Basil Kerski, Andrzej Kotula, Krzysztof Ruchniewicz, Kazimierz Wóycicki, Wrocław 2009, 173 ff.

${ }^{40}$ Vgl. z. B. Hilfe für Kinder Volkspolens, in: Neue Zeit, 18 XII 1981, 1; Aufruf zur Hilfe für VR Polen, in: Sächsisches Tageblatt, 18 XII 1981, 1.

${ }^{41}$ Wojtaszyn, Akcja pomocy NRD, aaO (Anm. 39), 179.

KZG/CCH 24 (2011), S. 1-10, ISSN 0932-9951

(c) Vandenhoeck \& Ruprecht GmbH \& Co. KG, Göttingen 2012
} 
der DDR sollte als ein Sinnbild der Unterstützung für General Wojciech Jaruzelski und seine Equipe betrachtet werden.

In diesem Zusammenhang hatte die Aktion „Hilfe für die Volksrepublik Polen" auch andere Bedeutung für SED-Propagandisten. Sie sollte ein Gegengewicht gegenüber den karitativen Maßnahmen darstellen, die in der Bundesrepublik stattfanden.

Die Hilfsaktion wurde seitens der DDR-Bürgern positiv aufgenommen. Breite Kreise der DDR-Bevölkerung, von altruistischen Gefühlen und Mitleid angetrieben, nahmen gerne an der Hilfe für die östlichen Nachbarn teil. Die positiven Reaktionen beweisen auch Berichte der Stasi ${ }^{42}$, die die ganze Aktion überwachte. Die Gründe der Teilnahme der DDR-Bürger an der „Hilfe für Volkspolen“ kann man auch als ein Art der „Belohnung“ für Polen interpretieren, die eigene Probleme mit eigenen zu Kräften lösen, ohne Notwendigkeit der militärischen Intervention der Warschau-Pakt-Armeen (und unter ihnen auch der Nationalen Volksarmee der DDR), was zu den größten Befürchtungen der DDR-Bürger in der Solidarność-Ära gehörte. ${ }^{43}$

Die deutschen Hilfsaktionen in den 1980er Jahren (sowohl aus der Bundesrepublik als auch der DDR) kann man als ersten wirklich großen psychologischen Durchbruch im Verhältnis zwischen Deutschen und Polen bezeichnen. ${ }^{44}$ Zweifelsohne war es einer der Meilensteine der späteren Versöhnung und Verständigung zwischen den im demokratischen Land lebenden Polen und den im vereinigten Land lebenden Deutschen. Es handelte sich in diesem Fall nicht nur um Worte, sondern um konkrete Taten.

\footnotetext{
${ }^{42}$ BStU MfS ZAIG 4152, 50, 58; BStU MfS HA VII 2950/Bd. 4, 24, 29, 34, 58; BStU MfS HA XX/AKG 5616, 132-133.

${ }^{43}$ Vgl. Zenobiusz Kozik, Niemcy w NRD a polskie kryzysy 1956 i 1980-1981, Piotrków Trybunalski 1998, 190; Ludwig Mehlhorn, Zwangsverordnete Freundschaft? Die Entwicklung der Beziehungen zwischen der DDR und Polen 1949-1990, in: Wolf-Dieter Eberwein/Basil Kerski (Hgg.), Die deutsch-polnischen Beziehungen 1949-2000. Eine Werte- und Interessengemeinschaft?, Opladen 2001, 69; Wojciech Wieczorek/Artur Hajnicz/Ludwig Mehlhorn/Timothy Garton Ash, Solidarność 1980/81 und die Folgen in Polen, in den sozialistischen Nachbarländern, insbesondere in der DDR, sowie im OstWest-Verhältnis, in: Materialien der Enquete-Kommission „Aufarbeitung von Geschichte und Folgen der SED-Diktatur in Deutschland“ (12. Wahlperiode des Deutschen Bundestages), hrsg. v. Deutschen Bundstag, Band V/1, Baden-Baden 1995, 209.

${ }^{44}$ Vgl. Adam Krzemiński, Vorwort, in: Riechers, Hilfe für Solidarność, aaO. (Anm. 15), 6.
}

KZG/CCH 24 (2011), S. 1-10, ISSN 0932-9951

(c) Vandenhoeck \& Ruprecht GmbH \& Co. KG, Göttingen 2012 
Dr. Dariusz Wojtaszyn, wissenschaftlicher Mitarbeiter am Willy-BrandtZentrum für Deutschland- und Europastudien der Universitaet Wroclaw, Ul. Strażnicza 1-3, 50-206 Wroclaw

KZG/CCH 24 (2011), S. 1-10, ISSN 0932-9951

(c) Vandenhoeck \& Ruprecht GmbH \& Co. KG, Göttingen 2012 